\title{
Delayed Abdominal Pseudocyst after Ventriculoperito- neal Shunt Surgery: A Case Report
}

\author{
Soung Bin Yim, Yeon Gu Chung, Yu Sam Won \\ Department of Neurosurgery, Kangbuk Samsung Hospital, Sungkyunkwan University School of Medicine, Seoul, \\ Republic of Korea
}

Corresponding author: Yu Sam Won

Department of Neurosurgery, Kangbuk Samsung Hospital, Sungkyunkwan University School of Medicine, 29 Saemunan-ro, Jongno-gu, Seoul 03181,

Republic of Korea

Tel: +82-2-2001-2450

Fax: +82-2-2001-2157

E-mail: yusam.won@samsung.com

Received: August 20, 2018

Revised: August 28, 2018

Accepted: August 29, 2018

\begin{abstract}
A 59-year-old man, who had a ventriculoperitoneal shunt (VPS) operation for the treatment of hydrocephalus due to cysticercosis 26 years prior, visited our hospital with increasing abdominal distention. An abdominal computed tomography (CT) scan showed well enhanced demarcated fluid collection about $20 \times 18 \mathrm{~cm}$ around the distal catheter tip. We performed exploratory laparoscopy and inserted a drainage catheter into the abdominal cyst. All cyst fluid analyses and cultures were negative. Over the course of the next eight weeks, abdominal CT demonstrated that the pseudocyst decreased. Following removal of the drainage catheter, abdominal $C T$ showed that the pseudocyst increased again. A drainage catheter was re-inserted and Taurolin ${ }^{\circledR}$ (Taurolidine) solution irrigation was performed for 3 months. Upon the completion of this 3 months, the patient complained of abdominal distension and abdominal CT found both that the pseudocyst was increased and doubted cystic infection. Eventually, an exploratory laparotomy and cyst resection were performed. Although the occurrence of pseudocyst following VPS surgery is a rare complication, it can cause additional complications such as obstruction and infection. If a patient has gastrointestinal symptoms, pseudocyst should be considered as one of the complications of VPS surgery.
\end{abstract}

Key Words: Cysticercosis; Cysts; Hydrocephalus; Intraabdominal infections; Ventriculoperitoneal shunt

\section{INTRODUCTION}

Ventriculoperitoneal shunt (VPS) is commonly used to treat hydrocephalus. There are a number of complications associated with VPS, such as shunt obstruction, shunt fracture, disconnection, migration, misplacement, over-drainage, and abdominal complications ${ }^{3)}$. One of the complications, abdominal pseudocyst, refers to localized fluid collection around the distal catheter surrounded by non-epithelium cell ${ }^{9}$. The purpose of this case is to report a delayed abdominal pseudocyst in patients with cysticercosis after VPS.

\section{CASE REPORT}

Our patient was a 59-year-old man, who had a VPS operation (PS Medical $^{\circledR}$ medium pressure valve; Medtronic, Minneapolis, MN, USA) for the treatment of hydrocephalus due to cysticercosis 26 years prior to visiting our hospital. About seven years prior, he reported having felt increasing abdominal distention, however he did not visit any hospital. About 1 week prior, he was admitted to the emergency department of another hos- pital with increased abdominal distention. Abdominal computed tomography (CT) scan and brain CT scan were performed in the other hospital's emergency department, then the patient was transferred to our hospital. Abdominal CT scan showed well enhanced demarcated fluid collection about $20 \times 18 \mathrm{~cm}$ around the distal catheter tip with no evidence of abdominal comorbidities (Fig. 1). The hydrocephalus was observed on
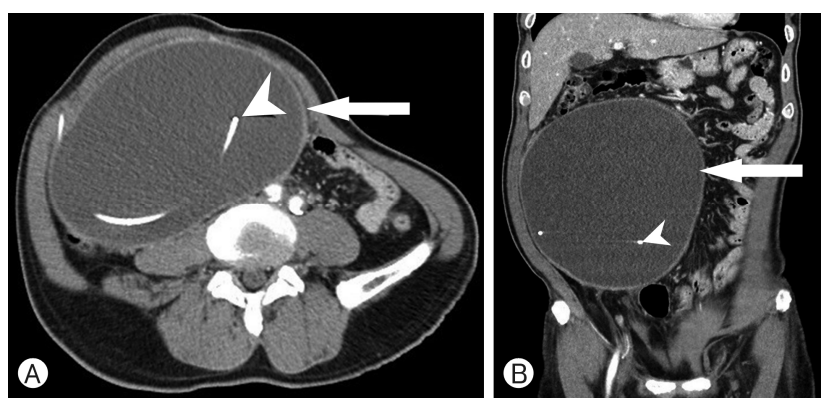

Fig. 1. Initial abdominal computed tomography $(C T)$, $(A)$ axial view of abdominal CT, (B) coronal view of abdominal CT. A well-demarcated fluid collection (white arrow) enhancing wall about $20 \times 18 \mathrm{~cm}$ on the right lower quadrant abdomen area. A distal tip of the shunt catheter (white arrowhead) is observed in the pseudocyst. 
initial brain CT scan (Evans index, 0.31) (Fig. 2). In addition, the patient did not exhibit any neurological symptoms (Glasgow
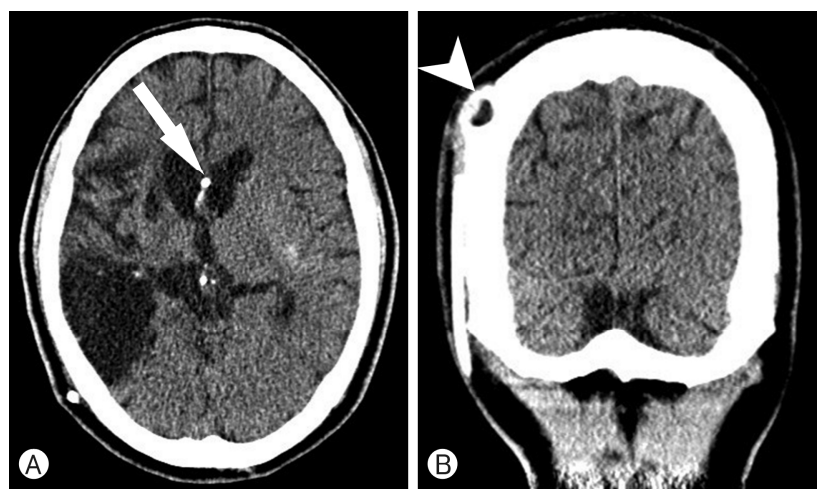

Fig. 2. Initial brain computed tomography (CT), (A) axial view of brain CT, (B) coronal view of brain CT. A distal catheter of ventriculoperitoneal shunt (VPS) was observed in the ventricle (white arrow). The reservoir of the VPS was located outside the skull (white arrowhead).
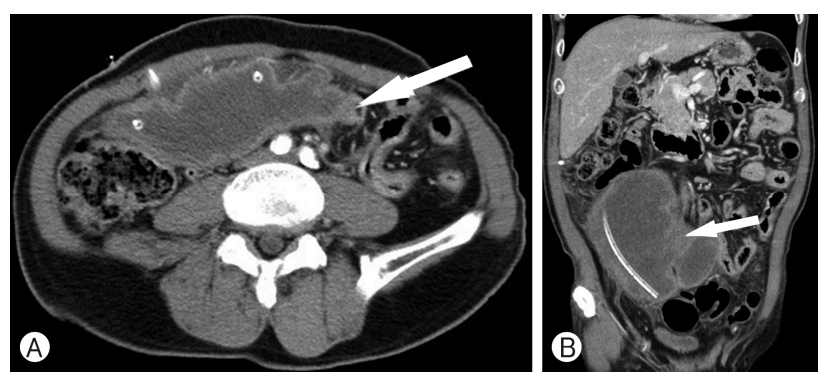

Fig. 3. Follow-up abdominal computed tomography (CT) after 8 weeks, (A) axial view of abdominal CT, (B) coronal view of abdominal CT. Significantly decreased size of fluid collection $(20 \times 18 \mathrm{~cm}$ to $13 \times 13 \mathrm{~cm})$ in the abdominal cavity. Pseudocyst was slightly improved compared to before but still shows wall enhancement and irregular thickening (white arrow).
Coma Scale, E4V5M6) and laboratory examinations showed no any abnormalities beyond elevated serum C-reactive protein (CRP; $3.10 \mathrm{mg} / \mathrm{dL}$ ). Serum immunoglobulin E level was normal as well $(88.6 \mathrm{kU} / \mathrm{L})$. Also, the result of specific serum immunoglobulin $\mathrm{G}$ (IgG) antibody of cysticercosis was negative.

We performed the exploratory laparoscopy and drainage catheter insertion into the abdominal cyst with a general surgery team after reposition of the distal shunt catheter. The cerebrospinal fluid (CSF) analysis from distal tip and abdominal cystic fluid analysis from pseudocyst were performed. The pseudocyst fluid was of a chocolate color. Cytology studies, however, were negative for malignant cells. Additionally, the CSF fluid and abdominal cystic fluid culturing were performed. However, the results of culturing were negative. An India ink test and crypto antigen test was also negative. Polymerase chain reaction for Mycobacterium tuberculosis was negative as well. After the placement of a drain to abdominal cyst, the drain continued to produce up to about 30 to $40 \mathrm{cc}$ of serosanguineous fluid each day. Anthelmintics were not used because specific IgG antibody of cysticercosis in serum and abdominal cystic fluid were negative level. Over the course of the next eight weeks, abdominal CT demonstrated that the pseudocyst decreased (Fig. 3).

Following drainage catheter removal, pseudocyst was increased again on abdominal CT. A catheter drainage re-insertion and Taurolin ${ }^{\circledR}$ (Taurolidine; Samjin Pharmaceutical Co., Seoul, Korea) solution irrigation (100 cc at once every week) were performed for 3 months, after which pseudocyst size was improved. Three months later, the patient complained of fever, abdominal pain and distension with abdominal tenderness. Follow up abdominal CT showed both that pseudocyst was increased and doubted cystic infection (Fig. 4). The drainage fluid has foul odor and infection was suspected in blood test included white blood cell count $\left(12,380 / \mathrm{mm}^{3}\right), \mathrm{CRP}(17.54 \mathrm{mg} / \mathrm{dL})$, and erythrocyte sedimentation rate $(70 \mathrm{~mm} / \mathrm{h})$. An exploratory laparotomy
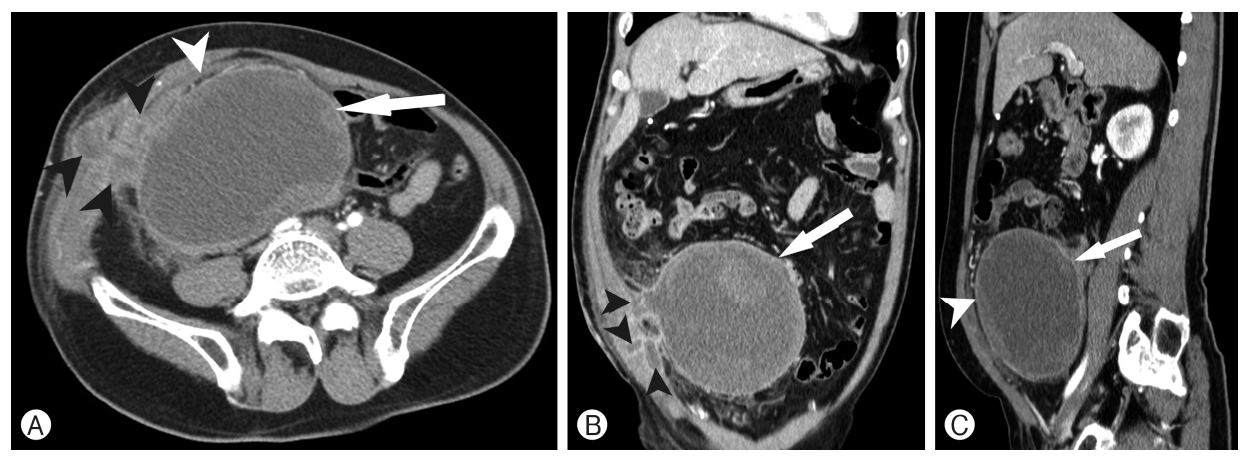

Fig. 4. Follow-up abdominal computed tomography $(\mathrm{CT})$ prior to exploratory laparotomy and cyst resection (8 months prior to initial), (A) axial view of abdominal CT, (B) coronal view of abdominal CT, (C) sagittal view of abdominal CT. Increased extent of intra-abdominal loculated fluid collection (11.8× $10.6 \mathrm{~cm}$ to $14.4 \times 10.5 \mathrm{~cm}$ ) with wall thickness and enhancement (white arrow). A newly seen multifocal small fluid collection with peripheral enhancement in the right abdominal wall is observed along the previous percutaneous catheter drainage insertion tract (black arrowhead). There is increased haziness and infiltration in the right omentum and peritoneum (white arrowhead). 
and cyst resection were performed by a general surgery team. Cystic mass was filled with pus-like fluid and had extreme tissue adhesion with peritoneum, omentum, cecum, and small bowel (Fig. 5). Pathology of biopsy revealed the fibrocollagenous tissue with chronic active inflammation, necrosis, abscess formation, and dystrophic calcification on focal findings (Fig. 6). The Methicillin-sensitive Staphylococcus aureus (MSSA) strain was cultured from pus-like fluid. Triaxone ${ }^{\circledR}$ (Ceftriaxone; Hanmi Pharmaceutical Co., Ltd., Seoul, Korea) 2 g intravenous injection (IV) and Trizele ${ }^{\circledR}$ (Metronidazole; JW Pharmaceutical., Seoul, Korea) $1.5 \mathrm{~g}$ IV were used as initial antibiotics after surgery and Cefazoline ${ }^{\circledR}$ (Cefazolin; Chong Kun Dang Pharmaceutical Co., Seoul, Korea) 6 g IV was used for 7 days after MSSA was cultured. After discharge, Amoxclan Duo ${ }^{\circledR}$ (Amoxicillin and

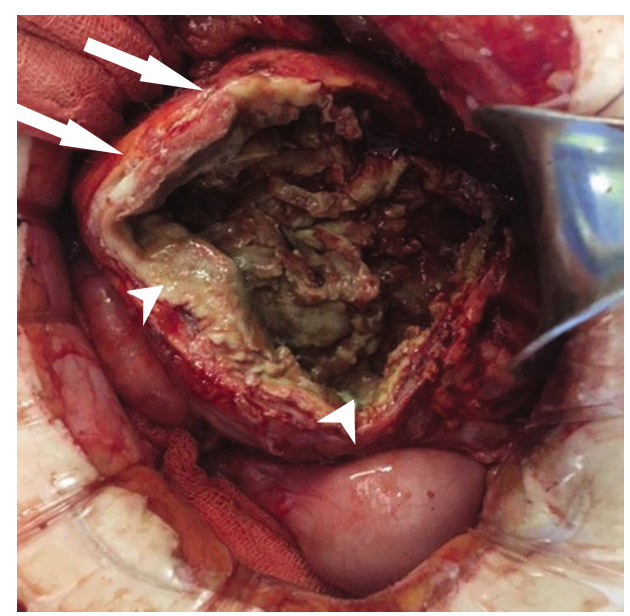

Fig. 5. An intra-operative view of pseudocyst. A pseudocyst with a yellowish-gray thick and irregular membrane (white arrow) of about $15 \times 15 \mathrm{~cm}$ was observed, and there are extreme adhesions surrounding tissues such as the peritoneum, omentum, cecum, and small bowel. When the incision was made on the anterior wall of the pseudocyst, a pus-like fluid (white arrowhead) leaked out. After the anterior wall was removed, the internal tissue was scraped off. A part of the posterior wall was left due to extreme adhesions of the surrounding tissues.
Clavulanic Acid; Hanmi Pharmaceutical Co.) $2 \mathrm{~g}$ per oral was used for 3 weeks. Thereafter, we have continued to observe him for 13 months, and he has been without further symptoms to this point.

\section{DISCUSSION}

VPS is a commonly accepted surgical technique for the treatment of hydrocephalus. VPS has several associated complications, with rates as high as $40 \%$ by 1 year and $50 \%$ by 2 years ${ }^{4)}$. The most common complications of VPS are obstruction, infection, and mechanical failure ${ }^{3)}$. Early shunt malfunctions are usually caused by misplacement, disconnection, or migration of the shunt components. The delayed complications of VPS are shunt fracture, over-drainage, loculation of the ventricles, and abdominal complications ${ }^{3}$.

Abdominal associated complications are ascites, pseudocyst, perforations, etc., while common symptoms of shunt malfunction are nausea, vomiting, irritability, fever, or an altered level of consciousness ${ }^{4}$. Neurologic examination can identify papilledema, cranial nerve palsies, hyperactive reflexes, and ataxic gait. Abdominal palpation is useful in confirming ascites or an intra-abdominal mass-like lesion. In this case report, the patient presented with increased abdominal distention and abdominal cyst was discovered on the abdominal CT scan. Pseudocysts, which are cases of loculated intra-abdominal fluid collection around the peritoneal catheter, were first reported by $\mathrm{Harsh}^{5)}$ in 1954 . Abdominal pseudocyst formation occurs in $0.33 \%$ to $6.8 \%$ of shunt procedures due to abdominal infection $^{6,8)}$. Pseudocysts are more common than ascites, and they present as a localized abdominal mass. Thus, patients with pseudocysts sometimes complain of abdominal discomfort and diffuse abdominal tenderness. If the pseudocyst is large, symptoms such as bowel obstruction may appear ${ }^{2)}$.

The exact pathogenesis of abdominal pseudocyst remains unknown ${ }^{6}$. Nevertheless, three causes are considered: (1) Chronic infection; (2) Particle like protein in CSF; and (3) Foreign body reaction. Tomiyama et al. ${ }^{10)}$ proposed that the development of
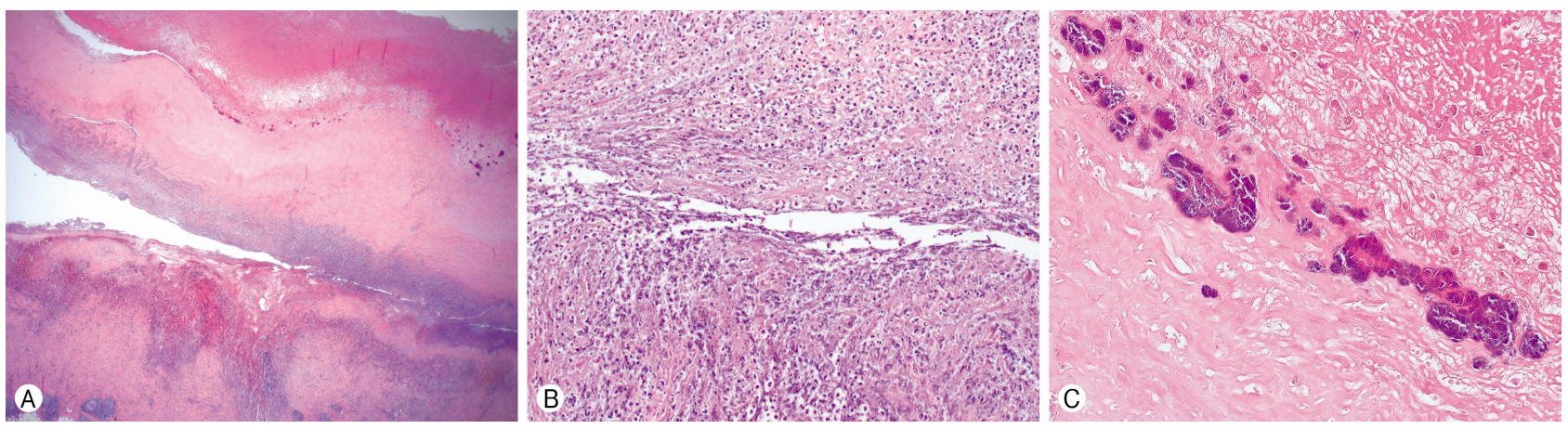

Fig. 6. A pathology of specimen. (A) A slide of typical cross-section (hematoxylin and eosin stain [H\&E], $\times 40$ magnification). (B) A slide of chronic active inflammation and necrosis $(H \& E, \times 200$ magnification). (C) A slide of dystrophic calcifications (H\&E, $\times 200$ magnification). 
pseudocyst suggests the presence of a chronic low-grade infection. However, it is not uncommon to find sterile fluid within the pseudocyst cavity when it is aspirated. Likewise, there is no evidence of any infection in this case report. In the case of children, patients with brain tumors who have VPS placed are more likely to develop pseudocysts, perhaps because of the elevated protein in the CSF interfering with abdominal absorption $^{1,2)}$. According to Pinto and de Oliveira ${ }^{7}$, VPS catheter is a foreign body that can cause infection or other foreign body reactions, resulting in epithelial responses which may lead to inflammation and obstruction. Other causes (such as a history of abdominal operations, abnormalities of the ascites, and silicon allergies) have also been reported.

Our adult case exhibited onset 26 years after VPS surgery. This case is considered to be an extremely a rare case of abdominal pseudocyst, because abdominal pseudocyst typically occurs 3 weeks to 5 years after VPS surgery in younger patients). Thus, it is important to consider an abdominal pseudocyst in adults as a candidate cause of acute malfunction of a VPS system, even when more than 5 years have passed since the operation. Also, more aggressive treatment such as direct cyst removal was needed, because keeping the drainage catheter for a long time was cause of the infection.

\section{CONCLUSION}

Although the occurrence of pseudocyst after VPS surgery is a rare complication, it can cause additional complications such as obstruction and infection. If patient has gastrointestinal symptoms, pseudocyst should be considered as one of the possible complications of VPS surgery. Even in the case that the patient reports no major problems for a long time after surgery, complications may still occur, even after over 20 years as in our case report, so periodic examination and observation are needed.

\section{CONFLICT OF INTEREST}

No potential conflict of interest relevant to this article was reported.

\section{REFERENCES}

1. Awori J, Wu CY, Maher CO: Malignant cause of ventriculoperitoneal shunt 'pseudocyst': a case report. Pediatr Neurosurg 50:73-75, 2015

2. Browd SR, Gottfried ON, Ragel BT, Kestle JR: Failure of cerebrospinal fluid shunts: part II: overdrainage, loculation, and abdominal complications. Pediatr Neurol 34:171-176, 2006

3. Browd SR, Ragel BT, Gottfried ON, Kestle JR: Failure of cerebrospinal fluid shunts: part I: Obstruction and mechanical failure. Pediatr Neurol 34:83-92, 2006

4. Garton HJ, Kestle JR, Drake JM: Predicting shunt failure on the basis of clinical symptoms and signs in children. J Neurosurg 94:202-210, 2001

5. Harsh GR, 3rd: Peritoneal shunt for hydrocephalus, utilizing the fimbria of the fallopian tube for entrance to the peritoneal cavity. J Neurosurg 11:284-294, 1954

6. Mobley LW, 3rd, Doran SE, Hellbusch LC: Abdominal pseudocyst: predisposing factors and treatment algorithm. Pediatr Neurosurg 41:77-83, 2005

7. Pinto FC, de Oliveira MF: Laparoscopy for ventriculoperitoneal shunt implantation and revision surgery. World J Gastrointest Endosc 6:415-418, 2014

8. Rainov N, Schobess A, Heidecke V, Burkert W: Abdominal CSF pseudocysts in patients with ventriculo-peritoneal shunts. Report of fourteen cases and review of the literature. Acta Neurochir (Wien) 127:73-78, 1994

9. Tamura A, Shida D, Tsutsumi K: Abdominal cerebrospinal fluid pseudocyst occurring 21 years after ventriculoperitoneal shunt placement: a case report. BMC Surg 13:27, 2013

10. Tomiyama A, Harashina J, Kimura H, Ito K, Honda Y, Yanai $\mathrm{H}$, et al.: An Intra-Abdominal Pseudocyst around a Ventriculoperitoneal Shunt due to Streptococcus Infection 7 Years after Shunt Surgery. Surg Res Pract 2014:898510, 2014 